\title{
Toward the Generation of Magnetized Collisionless Shocks with High-Power Lasers*)
}

\author{
Yoshitaka SHOJI, Ryo YAMAZAKI, Sara TOMITA, Yushiro KAWAMURA, Yutaka OHIRA, \\ Satoshi TOMIYA, Yoichi SAKAWA ${ }^{1)}$, Takayoshi SANO ${ }^{1)}$, Yukiko HARA ${ }^{1)}$, Sarana KONDO ${ }^{1)}$, \\ Hiroshi SHIMOGAWARA $^{1)}$, Shuichi MATSUKIYO ${ }^{2)}$, Taichi MORITA ${ }^{2)}$, Kentaro TOMITA $^{2)}$, \\ Hitoki YONEDA $^{3)}$, Kazunori NAGAMINE ${ }^{3)}$, Yasuhiro KURAMITSU ${ }^{4)}$, Toseo MORITAKA ${ }^{4)}$, \\ Naofumi OHNISHI ${ }^{5)}$, Takayuki UMEDA $^{6)}$ and Hideaki TAKABE ${ }^{7)}$ \\ Department of Physics and Mathematics, Aoyama Gakuin University, 5-10-1 Fuchinobe, Sagamihara 252-5258, Japan \\ ${ }^{1)}$ Institute of Laser Engineering, Osaka University, Suita 565-0871, Japan \\ ${ }^{2)}$ Department of Earth System Science and Technology, Kyushu University, Kasuga 816-8580, Japan \\ ${ }^{3)}$ Institute for Laser Science, University of Electro-Communications, Chofu 182-8585, Japan \\ ${ }^{4)}$ Department of Physics, National Central University, Taiwan \\ ${ }^{5)}$ Department of Aerospace Engineering, Tohoku University, Sendai 980-8579, Japan \\ ${ }^{6)}$ Institute for Space-Earth Environmental Research, Nagoya University, Nagoya 464-8601, Japan \\ 7) Dresden Center, Helmholtz, Society, Germany
}

(Received 8 January 2016 / Accepted 12 February 2016)

\begin{abstract}
We have started a project of experimental generation of low Mach number collisionless shocks propagating into magnetized plasma, using high power lasers such as Gekko XII at Institute of laser engineering, Osaka University. We briefly present a result of our first experiments done in September 2014, in which Aluminum plane target was irradiated by main laser in ambient Helium gas with external magnetic field. Ejected Aluminum plasma had a bulk velocity of $430 \mathrm{~km} \mathrm{~s}^{-1}$, which is sufficient enough to excite the magnetized collisionless shock in our experimental setup. However, the ejected Aluminum plasma was decelerated before the shock was excited.

(C) 2016 The Japan Society of Plasma Science and Nuclear Fusion Research
\end{abstract}

Keywords: laboratory astrophysics, supernova remnant shock, magnetized collisionless shock, particle acceleration, cosmic ray

DOI: $10.1585 /$ pfr. 11.3401031

\section{Inroduction}

Collisionless shocks are ubiquitous in various astrophysical, heliospheric, solar-terrestrial, and laboratory phenomena. The injection problem in diffusive shock acceleration (DSA) at the collisionless shocks is one of the most important outstanding issues. DSA, or first order Fermi acceleration, has been regarded as the most plausible acceleration mechanism of galactic cosmic rays [1]. It is assumed in the DSA model that non-thermal charged particles can traverse a shock back and forth by interacting with turbulent plasma waves upstream and downstream of the shock. The upstream turbulent medium flows faster into the shock and the downstream one flows more slowly away from the shock, so that the particles are scattered by the converging upstream and the downstream waves and statistically gain energy. In this model, the pre-existence of the non-thermal particles is assumed. However, it is not trivial how and what amount of those non-thermal particles are produced.

Supernova remnant shocks, which are representative

author'se-mail: shoji@phys.aoyama.ac.jp,ryo@phys.aoyama.ac.jp

*) This article is based on the presentation at the Conference on Laser Energetics 2015 (CLE2015). of high-energy astrophysical phenomena, produce highenergy cosmic rays at least up to $10^{14} \mathrm{eV}$. This fact has been confirmed by observations of their X-and gammaray emission [2,3]. Interestingly, an extremely high injection rate, corresponding to a cosmic-ray momentum flux of more than $50 \%$ of the gas pressure in the shock downstream region is implied, although not yet conclusive [4]. If this is the case, the back-reaction of high-energy particles is so significant that the shock structure itself as well as wave excitation processes are modified. However, since these facts are implied by remote sensing, we cannot directly observe the precise mechanism of the acceleration mechanism.

Numerical simulation using recent high performance computers is also a powerful tool to investigate the injection processes, although this tool is also not perfect. The hybrid and full particle-in-cell (PIC) simulations can reproduce the self-consistent structure of a shock including the effects of wave-particle interactions that should be essential for the injection processes. Detailed wave excitation processes as well as the associated acceleration processes of ions and electrons have been discussed [5,6]. According to these simulation studies, the injection processes are 
complex and dominated by a variety of multi-scale phenomena that vary with parameters. This implies the importance of measuring the macro and the micro scale shock structures simultaneously in observations. Currently, on the other hand, the so-called global simulation with realistic parameters has not been successful due to the limitation of computer resources even with lower cost hybrid simulations $[7,8]$. As a result, there is still a non-negligible gap between simulation results and observations.

Recently, laboratory astrophysics has developed, reproducing a collisionless shock in the laboratory (e.g., [9-11]). Both the macro and the micro scale structures of a shock are in principle simultaneously accessible. Moreover, physical parameters of a shock in a laboratory are controllable. For instance, the Alfvén Mach number can be controlled by changing the strength of an external magnetic field. Although this potential ability of the laboratory experiment is attractive, the methodology of experiments or the data analysis techniques have not been well established so far. Now, we will probe the needs for laboratory experiments to be new basic tools in the collisionless shock physics.

\section{Experiment}

Using Gekko-XII HIPER laser system (wavelength $352 \mathrm{~nm}$, pulse duration $500 \mathrm{ps,} \mathrm{energy} \sim 120 \mathrm{~J}$ per beam, and focal spot diameter $\sim 300 \mu \mathrm{m}$ ) at Institute of Laser Engineering, Osaka University, we made an experiment of the generation of low Mach number collisionless shocks propagating into magnetized plasma. In order to excite the collisionless shock in magnetized plasmas, an Aluminum plane target was irradiated. A schematic side view of the target and laser configuration is shown in Fig. 1. Before the experiment, we performed one-dimensional radiation hydrodynamics simulation, using ILESTA-1D code, and computed the velocity and density of the ejected plasma to design the experimental setup. We found that the bestestimated target is $\mathrm{Al}$ foil with thickness of $5-15 \mu \mathrm{m}$. The plasmas were diagnosed by a streaked optical pyrometry (SOP) measuring the self- emission, whose intensity strongly depends on the electron density in optically thin plasmas produced in the experiment. The SOP is a streaked data of an one-dimensional image behind the plane target at a certain wavelength using a band-pass filter which has a central wavelength of $450 \mathrm{~nm}$. It measures from the transverse direction as shown in the top view of the target in Fig. 2.

\section{Result}

Here, we present a result of 3-beams shot (laser energy of $333 \mathrm{~J}$ in total) with Aluminum plane target $(5 \mu \mathrm{m}$ thickness) in He ambient gas (3.3 Torr). The field strength of external magnetic field is $B \sim 4 \mathrm{~T}$ at the target. Figure 3 shows a stack of line profiles taken from the SOP image at $2 N$ ns, where $N$ is an integer between -1 and 20 . For ease

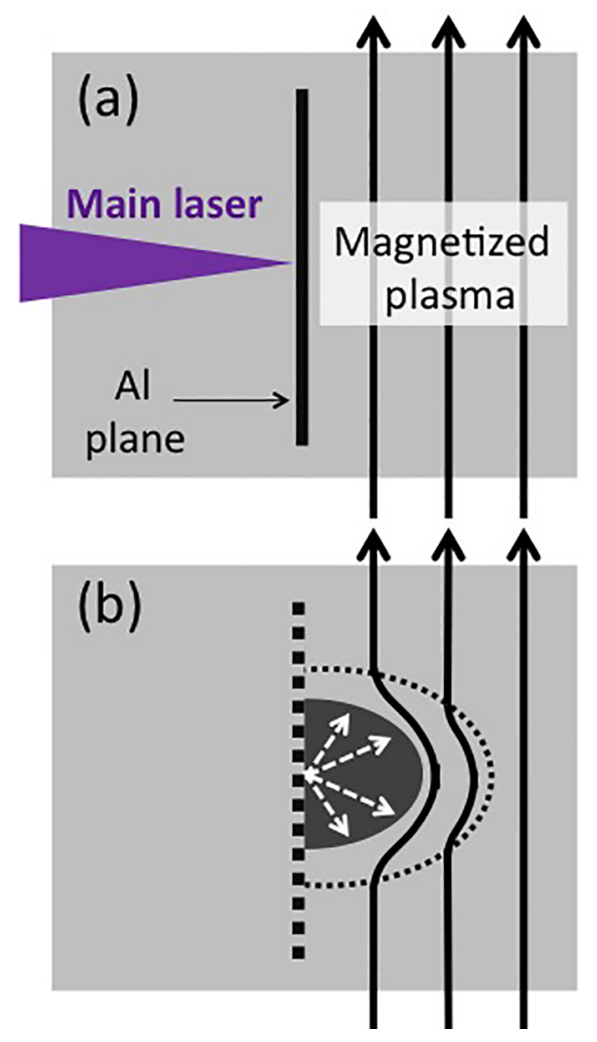

Fig. 1 (a) Schematic side view of Aluminum target and laser configuration. Before the shot, Helium ambient gas is filled with Chamber. At the moment of the shot, the external magnetic field is applied. The ambient gas is ionized by ionizing photons from Aluminum plane, becoming magnetized plasma. (b) The Helium plasma is pushed by Aluminum plasma (dark region). The magnetized shock is generated in the Helium plasma (dotted line).

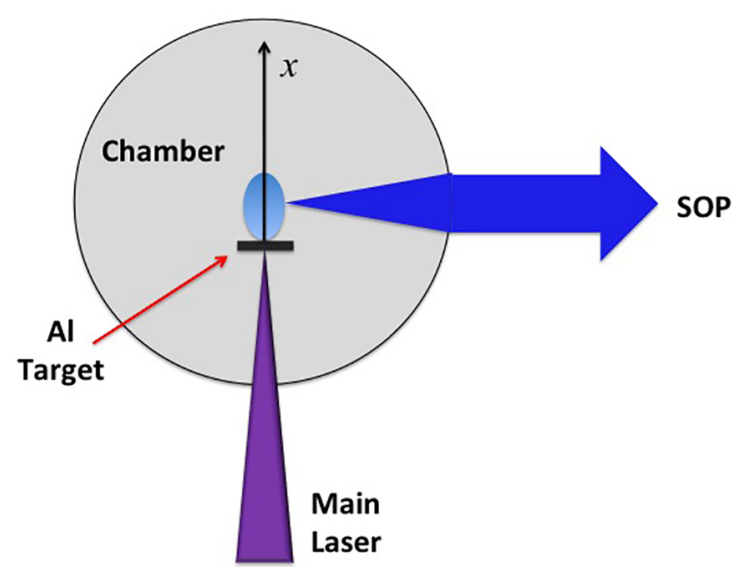

Fig. 2 The top view of the target. The plasmas are diagnosed by the SOP from the direction perpendicular to the plasma expansion. The $x$ axes is defined as the distance from the Al target along with the main laser.

of view, $1250(N+2)$ counts have been added at each time. We found that the ejecta velocity is $v_{\mathrm{ej}} \sim 430 \mathrm{~km} / \mathrm{s}$ until $8 \mathrm{~ns}$ after the shot. The flow velocity is consistent with the 


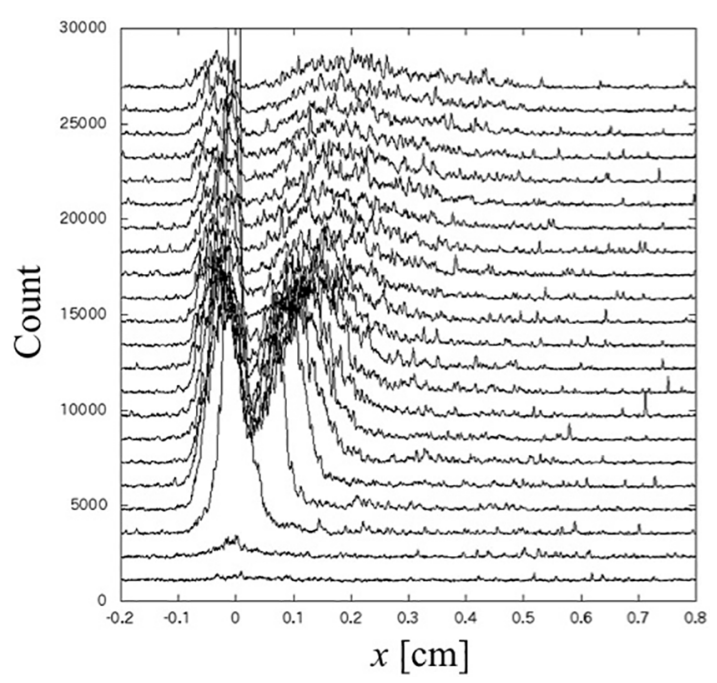

Fig. 3 Stack plot of line profiles along with the laser axis ( $x$ axis as shown in Fig. 2) of the SOP image from -2 to 40 ns. Initially Aluminum plane target was located at $x=0$. The main laser came from left to right.

result of one-dimensional radiation hydrodynamics simulation. As clearly seen, the ejecta plasma was decelerated after $8 \mathrm{~ns}$ due to an interaction with a He plasma.

Assuming that Aluminum atoms in the focal spot region form a high-velocity ejecta, we estimate the total mass of the Aluminum ejecta, $M_{\mathrm{Al}}=9.5 \times 10^{-7} \mathrm{~g}$. Then, the kinetic energy of Aluminum ejecta plasma is, $E_{\mathrm{K}} \sim(1 / 2) M_{\mathrm{Al}} v_{\mathrm{ej}}^{2} \sim 90 \mathrm{~J}$, which corresponds to $24 \%$ of the laser energy. If this energy were used to expel the ambient magnetic field, the magnetic stopping radius would be $\left(3 \mu_{0} E_{\mathrm{K}} / 2 \pi B^{2}\right)^{1 / 3}=1.5(B / 4 \mathrm{~T})^{-2 / 3} \mathrm{~cm}$. In our experiment, the ejecta plasma was decelerated before reaching this radius, so that some electrostatic interactions between Aluminum and Helium plasmas might occur.

\section{Discussion}

Ejecta velocity is high enough to excite a collisionless shock for our experimental conditions, because the ion-ion mean free path for He ions is estimated as $6.2 \mathrm{~cm}$ for ion velocity $v_{\text {ej }}=430 \mathrm{~km} / \mathrm{s}$ and the ion number density of $1.1 \times 10^{17} \mathrm{~cm}^{-3}$ that is expected for fully ionized $\mathrm{He}$ plasma. This length is larger than the system size. The velocity of $430 \mathrm{~km} / \mathrm{s}$ corresponds to the Alfvén Mach number, $M_{\mathrm{A}} \sim 3.3(B / 4 \mathrm{~T})^{-1}$, which can be larger than the critical Mach number. However, the ejecta was decelerated $8 \mathrm{~ns}$ after the shot whose mechanism is under investigation. Ion cyclotron period in He plasma is $16(B / 4 \mathrm{~T})^{-1}$ ns, so that the ejecta was decelerated before ions turned around due to the external magnetic field. Hence in future experiments, we need longer life time of the ejecta plasma without deceleration.

\section{Acknowledgment}

This work was performed under the joint research project of the Institute of Laser Engineering, Osaka University. This work was supported in part by grant-in-aid from the Ministry of Education, Culture, Sports, Science, and technology (MEXT) of Japan, No. 15K05088 (R.Y.) and 15H02154 (Y.S.).

[1] R. Blandford and D. Eichler, Phys. Rep. 154, 1 (1987).

[2] K. Koyama et al., Nature 378, 255 (1995).

[3] F. Aharonian et al., Nature 432, 75 (2004).

[4] E.A. Helder, J. Vink et al., Science 325, 719 (2009).

[5] R.A. Treumann, Astron. Astrophys. Rev. 17, 409 (2009).

[6] D. Burgess and M. Scholer, Space Sci. Rev. 178, 513 (2013).

[7] N. Omidi et al., J. Geophys. Res. 110, 12212 (2005).

[8] X. Blanco-Cano et al., J. Geophys. Res. 111, 10205 (2006).

[9] Y. Kuramitsu et al., Phys. Rev. Lett. 106, 175002 (2011).

[10] C. Niemann et al., Phys. Plasmas 20, 012108 (2013).

[11] C. Niemann, Geophys. Res. Lett. 41, 7413 (2014). 